Federal Reserve Bank of Minneapolis

Research Department Staff Report 126/JV

\title{
The Firm and the Plant in General Equilibrium Theory
}

\author{
Andreas Hornstein* \\ University of Western Ontario \\ Edward C. Prescott* \\ Federal Reserve Bank of Minneapolis \\ and University of Minnesota
}

\begin{abstract}
The general equilibrium formulations are developed for two important economic environments. The first environment is the Lucas managerial span-of-control theory of the firm. It is shown that, in the spirit of McKenzie, the aggregate production set can be characterized by a convex cone. The second environment permits both the number of hours plants are operated and the number of workers operating them to be varied. For empirically reasonable elasticities of substitution, equilibrium is characterized by employment-consumption lotteries.

*This paper was presented at the Conference on General Equilibrium and Growth: The Legacy of Lionel McKenzie, held May 5-7, 1989, at the University of Rochester. We would like especially to thank Lionel McKenzie and an anonymous referee for helpful comments. The views expressed herein are those of the authors and not necessarily those of the Federal Reserve Bank of Minneapolis or the Federal Reserve System.
\end{abstract}




\section{Introduction}

In this paper we introduce the firm and the plant into classical general equilibrium theory. Our approach follows McKenzie's in having a convex cone aggregate technology. McKenzie (1959) shows this formulation is equivalent to the one of Arrow and Debreu (1954), who assume a finite set of technologies and an ownership distribution. The nature of McKenzie's argument is that a distinct entrepreneurial factor can be introduced for each firm. In the Arrow-Debreu setup, the quantities of these factors are normalized to one and distributed proportionally to ownership shares. Both the McKenzie and the Arrow-Debreu formulations are equivalent mathematically. They are not, however, equivalent from the perspective of economics. The approach of identifying each firm with a distinct technology set is not at all useful for developing a theory of industrial organizations. As McKenzie (1959) points out, when nonconvexities are small relative to the size of the economy and access to the underlying technologies is free, the aggregate production possibility set is approximately a convex cone. In this paper, we adopt this approach and formally develop the theory of the aggregate production possibility set for two economies.

In Section 2, we represent the Lucas (1978) managerial span-of-control theory of the firm in the McKenzie-type general equilibrium language. With this representation the proof of existence and optimality of equilibrium is a simple application of standard theorems in general equilibrium theory. One element of this environment is a nonconvexity in the household consumption possibility set: An agent can either be a manager or be managed by someone else but cannot be one-third manager and two-thirds worker. This nonconvexity disappears with the introduction of lotteries: An agent can be a manager with probability one-third and a worker with probability two-thirds. This

is the approach that Prescott and Townsend (1984) use to extend competitive theory to a class of economies with private information. For the span-of-control economy, as well as for the PrescottTownsend private information economies, the production possibility set is a convex cone. 
In Section 3, we consider an environment in which both the length of time a plant can be operated and the number of workers operating it can be varied. This introduces what appears to be a nonconvexity in the production possibility set because a plant's output increases proportionally to hours of operation, given the number of workers operating it and the capital stock used by the workers. Until now, this feature has not been introduced into quantitative general equilibrium analyses. Here, using the abstract language of general equilibrium theory, we develop a simple, tractable way to introduce this feature. The important result is that in this world of identical individuals, some people work and some do not, even though preferences are convex. This result provides theoretical justification for the labor indivisibility constraint of Rogerson (1988) and Hansen (1985). Again, the production possibility set is a convex cone.

In Section 4, we use a theorem of McKenzie (1959) to establish the existence of a competitive equilibrium for the managerial span-of-control economy.

Throughout this paper, we use the McKenzie-type representation of an economy. A commodity point $\mathrm{x}$ is an element of a linear commodity space $\mathrm{L}$. There is a finite number of agent types, $i \in I$, and a continuum of each type. The measure of type $i$ is $\lambda^{i}$, and the total measure of agents in the economy is $\lambda=\Sigma_{i \in \lambda} \lambda^{i}$. The consumption set $X^{i}$ of a type $i$ agent is a subset of the commodity space $\mathrm{L}$. Preferences over consumption bundles in $\mathrm{X}^{\mathrm{i}}$ are represented by the utility functions $u^{i}: X^{i} \rightarrow \mathbf{R}$. Production is described by some aggregate production possibility set $Y$, which is a convex cone in $L$. An allocation $\left[\left(x^{i}\right)_{i \in I}, y\right]$ is feasible if $x^{i} \in X^{i}$ for all $i \in I, y \in Y$, and $\Sigma_{\mathrm{i} \in \mathrm{I}} \lambda^{\mathrm{i}} \mathrm{x}^{\mathrm{i}}=\mathrm{y}$. Endowments are subsumed in $\mathrm{X}^{\mathrm{i}}$. An economy is thus completely described by $\mathrm{E}=$ $\left\{\left(u^{i}, X^{i}, \lambda^{i}\right)_{i \in 1}, Y\right\} .^{1}$ 


\section{The Firm in General Equilibrium Theory}

In a paper on the distribution of firm size, Lucas (1978) describes a static model of entry and exit with competition in which firms arise endogenously. ${ }^{2}$ His model is a general equilibrium model, but his representation of the environment is such that its relationship to the standard general equilibrium formulation is not apparent. We show that by defining the commodity points in terms of contracts with lotteries, we can represent his environment as an economy whose production set is a convex cone. This formulation makes transparent the role of entrepreneurial ability as a production factor. It allows us to use standard existence results from general equilibrium theory, and it provides an example for McKenzie's interpretation of the aggregate production set.

We describe the environment of this economy as follows. There is a continuum of agents. Each agent is characterized by managerial ability, $m \in M$, and by endowment of the homogeneous capital good, $k \in K$. There is a finite number of agent types, $i \in I$. Each type is characterized by the pair $\left(\mathrm{k}^{\mathrm{i}}, \mathrm{m}^{\mathrm{i}}\right)$. The measure of agent type $\mathrm{i}$ is $\lambda^{\mathrm{i}}$ and $\Sigma_{\mathrm{i}} \lambda^{i}=1$. A homogeneous consumption good is produced using managerial ability, labor, and capital. The technology is such that if one agent of ability $m \in M$ manages $n \in N$ workers and $k \in K$ units of capital, then $m g[f(k, n)]$ units of the consumption good can be produced by this firm. The function $f$ displays constant returns to scale. Function $g$ is increasing, differentiable, and strictly concave with $g^{\prime}(\infty)=0$ and $g^{\prime}(0)=\infty$. The vector $\mathrm{a}=(\mathrm{k}, \mathrm{m}, \mathrm{n}) \in \mathrm{A}=\mathrm{K} \times \mathrm{M} \times \mathrm{N}$ denotes a particular productive activity.

Preferences with respect to random consumption allocations with support $\mathbf{C} \subset \mathbf{R}_{+}$are represented by their expected utility $\mathrm{E}[\mathrm{U}(\mathrm{c})]$. The function $\mathrm{U}$ is increasing and has a strictly concave extension on $\mathbf{R}_{+}$. An agent can act as either a manager or a worker, but not as both simultaneously.

Let the sets $C, K, M$, and $N$ be finite subsets of $R$, and let $q$ be the cardinality of $S=C \times$ $K \times(M \cup\{0\})$. For this environment the commodity space $L$ is $\mathbf{R}^{\mathrm{q}}$. We restrict our attention to 
finite sets for ease of exposition. This does not imply any loss of generality, and we will relax this assumption when appropriate.

For an agent, the feasible consumption bundle is interpreted as a contract that specifies the probabilities $x_{2}$ of the events $s=(c, k, m) \in S .{ }^{3}$ If the realization of the lottery is $s$, the agent receives $c$ units of the consumption good and provides $\mathrm{k}$ units of capital services; if $\mathrm{m}=0$, provides one unit of labor services; or if $m \neq 0$, provides one unit of managerial services of type $m$. Let

$$
S^{i}=\left\{s \in S: k \leq k^{i}, \quad m=0 \text { or } m=m^{i}\right\}
$$

Given that the agent's endowment of managerial ability and capital is assumed to be verifiable, the consumption possibility set of a type $i$ agent is

$$
X^{i}=\left\{x \in L_{+}: \sum_{s} x_{3}=1, \quad x_{3}=0 \text { if } s \notin S^{i}\right\}
$$

Note that an agent's consumption possibility set is a lower-dimensional subspace of the commodity space. This is a nonstandard feature that our economy has in common with McKenzie's general equilibrium approach. Preferences with respect to elements of this set are represented by the utility function

$$
\mathrm{u}(\mathrm{x})=\sum_{\mathrm{c}, \mathrm{k}, \mathrm{m}} \mathrm{U}(\mathrm{c}) \mathrm{x}_{\mathrm{ckm}}=\sum_{s} \mathrm{U}(\mathrm{c}) \mathrm{x}_{\mathrm{s}}
$$

An allocation $\left(\mathrm{x}^{\mathrm{i}}\right)_{\mathrm{i} \in \mathrm{I}}$ is resource feasible if there exists a $z \in \mathbf{R}_{+}^{\ell}$, where $\ell$ is the cardinality of $\mathrm{A}$, such that the following constraints are met:

$$
\begin{aligned}
& \sum_{i} \lambda^{1} \sum_{s} c x_{z}^{i}-\sum_{i} \operatorname{mg}[f(k, n)] z_{a} \leq 0 \\
& -\sum_{i} \lambda^{i} \sum_{s} k x_{s}^{i}+\sum_{a} k z_{a} \leq 0 \\
& -\sum_{i} \lambda^{i} \sum_{c, k} x_{c k 0}^{i}+\sum_{a} n z_{a} \leq 0
\end{aligned}
$$


(6) $\quad-\sum_{i} \lambda^{i} \sum_{c, k} x_{c k m}^{i}+\sum_{k, \mathbf{n}} z_{k m n} \leq 0$, for each $m \in M$.

We assume that a law of large numbers holds such that the realized distribution on outcomes $s$ for an agent type $i$ coincides with that agent type's probability measure $x^{i}$ (see, for example, Uhlig 1988). The vector $z$ is a measure on the set of feasible activities a $\in A$. The measure $z$ specifies the activities internal to the production set.

Constraint (3) says the total quantity of the consumption good distributed is less than or equal to the total amount produced. Constraint (4) says the total quantity of capital provided by the agents is at least as great as the quantity used in production. Constraints (5) and (6) state the same about the production factors, labor $(\mathrm{m}=0)$ and managerial abilities ( $\mathrm{m} \in \mathrm{M}$ ).

The system of linear constraints (3)-(6) can be expressed in the following compact form

(7) $\quad r\left(\sum_{i} \lambda^{i} x^{i}, z\right) \leq 0$

where $\mathrm{r}$ is a linear function.

We now define the technology for this economy. The production set is

(8) $\quad \mathrm{Y}=\left\{\mathrm{y} \in \mathrm{L}_{+}\right.$: there exists $\mathrm{z} \in \mathbf{R}_{+}^{\ell}$ for which $\left.\mathrm{r}(\mathrm{y}, \mathrm{z}) \leq 0\right\}$

where $\mathbf{r}$ represents a finite number of linear constraints. As defined, the production set is McKenzie's convex cone. This general equilibrium formulation seems to provide no information about the industrial structure of the economy because the structure has been subsumed in the production set $\mathrm{Y}$. We can-and do-interpret a firm to be some combination of a manager, capital, and workers, and in equilibrium there will be some distribution $\mathrm{z}$ over firms. The formation of firms thus becomes part of the economy's production possibility set, not something that exists independently of it. 


\section{The Plant in General Equilibrium Theory}

Rogerson (1988) and Hansen (1985) have done important work on modeling employment in quantitative general equilibrium theory. They introduce a labor indivisibility constraint in the environments considered. In those environments, in which all individuals are identical, equilibrium is characterized by some people working and some not. We consider an environment in which the same employment pattern is observed, although hours of employment is not constrained to two values. In our environment both the time a plant can be operated and the number of workers operating a plant can be varied. This introduces what appears to be a nonconvexity in the production possibility set, since the output of a plant is $h f(k, n)$, where $h$ is hours the plant is operated, $f$ is a neoclassical production function with constant returns to scale, $\mathrm{k}$ is capital input, and $\mathrm{n}$ is the number of workers operating a plant. For this technology, we no longer assume that production uses two distinct inputs, managerial services and labor services, as in Section 2.

We now consider an economy of ex ante identical individuals, where the measure of agents is one. Preferences with respect to random consumption work pairs $(c, h)$ are represented by their expected utility $E[U(c, h)]$, where $c \geq 0$ is consumption, $0 \leq h \leq 1$ is the fraction of time allocated to market activities, and $1-h$ is the fraction of time allocated to nonmarket activities. Each agent is endowed with $\overline{\mathbf{k}}>0$ units of capital. The function $U$ is increasing in $c$ and decreasing in $h$.

The problem is to represent this economy in our McKenzie-type general equilibrium language. Let $\mathrm{C}, \mathrm{H}$, and $\mathrm{K}$ be finite sets, and let $\mathrm{S}$ be $\mathrm{C} \times \mathrm{H} \times \mathrm{K}$ with generic element $\mathrm{s}=$ $(c, h, k)$. The commodity space $L$ is the Euclidean space with dimension equal to the cardinality of the set $\mathbf{S}$. For an agent, the consumption bundle is interpreted as a contract that obliges the agent to provide $\mathrm{k}$ units of capital and $\mathrm{h}$ units of time, for which the agent receives $\mathrm{c}$ units of the consumption good. The probability of an event $s=(c, h, k)$ is $x_{s}$. There is only one type of agent with the consumption possibility set 
(9) $\mathrm{X}=\left\{\mathrm{x} \in \mathrm{L}_{+}: \sum_{\mathrm{s} \in \mathrm{S}} \mathrm{x}_{\mathrm{s}}=1\right.$, and $\mathrm{x}_{\mathrm{chk}}=0$ if $\left.\mathrm{k}>\overline{\mathrm{k}}\right\}$

and the utility function

$$
\mathfrak{u}(\mathbf{x})=\sum_{c, b, \mathbf{l}} \mathrm{U}(\mathrm{c}, \mathrm{h}) \mathrm{x}_{\mathrm{chk}}=\sum_{\mathbf{s}} \mathrm{U}(\mathrm{c}, \mathrm{h}) \mathrm{x}_{\mathbf{s}} .
$$

Let $\mathrm{N}$ be a finite set, and let $\mathrm{A}=\mathrm{H} \times \mathrm{K} \times \mathrm{N}$ with generic element $\mathrm{a}=(\mathrm{h}, \mathrm{k}, \mathrm{n})$ and cardinality $\ell$. We let $\mathrm{z}_{\mathrm{a}}$ be the number of plants operated $\mathrm{h}$ hours using $\mathrm{k}$ units of capital and $\mathrm{n}$ workers. An allocation is resource feasible if there exists some $\mathbf{z} \in \mathbf{R}_{+}^{\ell}$ such that

$$
\begin{aligned}
& \sum_{k} c x_{k}-\sum_{a} h f(k, n) z_{a} \leq 0 \\
& -\sum_{i} k x_{i}+\sum_{a} k z_{a} \leq 0 \\
& -\sum_{c, k} x_{c h k}+\sum_{k, n} n z_{h k n} \leq 0, \text { for all } h \in H .
\end{aligned}
$$

Constraint (11) says the amount of the consumption good distributed is less than or equal to the quantity produced. Constraint (12) says the quantity of the capital obtained is at least as great as the quantity of capital used in the production. Finally, constraints (13) state that the number of people working $\mathrm{h}$ hours are the numbers of people working in plants that are operated $\mathrm{h}$ hours.

Constraints (11)-(13) can be expressed in the compact form

$$
r(x, z) \leq 0
$$

and the technology set is

$$
Y=\left\{y \in L_{+}: \text {there exists } z \in \mathbf{R}_{+}^{\ell} \text { for which } r(y, z) \leq 0\right\}
$$


The constraints defining $\mathrm{Y}$ are jointly linear in $\mathrm{y}$ and $\mathrm{z}$. It is immediate that $\mathrm{Y}$ is a convex cone. The set $\mathrm{X}$ is convex, and the utility function $\mathrm{u}(\mathrm{x})$ is concave and continuous. Both sets are closed, and the set $\mathrm{X}$ is compact.

In this economy with one agent type, the anonymous Pareto-optimal allocation is the one that maximizes $\mathrm{u}(\mathrm{x})$ subject to $\mathrm{x} \in \mathrm{X} \cap \mathrm{Y}$. An optimum exists, given that $\mathrm{u}$ is continuous and $\mathrm{X} \cap \mathrm{Y}$ is compact. For economies with only one agent type, the anonymous competitive equilibrium and the anonymous Pareto-optimal allocations coincide.

The characteristics of an anonymous Pareto-optimal allocation are derived by analyzing a simpler equivalent problem. For this version, we no longer distinguish between the organization of consumption ( $\mathrm{x}$ ) and the organization of production (z). The event $s=(\mathrm{c}, \mathrm{h}, \mathrm{k}) \in \mathrm{S}$ is now interpreted as an agent receiving $c$ units of the consumption good, providing $h$ units of time, and working in a plant that uses $\mathrm{k}$ units of capital per worker. The Pareto-optimal allocation then solves the following linear program:

$$
\max _{x \geq 0} \sum_{s} U(c, h) x_{s}
$$

subject to the constraints

$$
\begin{aligned}
& \sum_{s} x_{s}=1 \\
& \sum_{s}[c-f(k, 1) h] x_{s} \leq 0 \\
& \sum_{s} k x_{s} \leq \bar{k} .
\end{aligned}
$$

Given that $f$ displays constant returns to scale, the solution to this problem has the same measure of agents consuming $\mathrm{c}$ units and working $\mathrm{h}$ hours in plants with $\mathrm{k}$ units of capital per worker as does the solution to the original problem. 
For the rest of this section, we choose standard functional forms for the utility and production function. The utility function is

$$
\mathrm{U}(\mathrm{c}, \mathrm{h})=\frac{\left[\mathrm{c}^{\gamma}(1-\mathrm{h})^{(1-\gamma)}\right]^{(1-\sigma)}-1}{(1-\sigma)}
$$

where $\gamma \in(0,1)$ and $\sigma \geq 0$. The production function is Cobb-Douglas,

$$
f(k, n)=k^{\alpha} n^{(1-\alpha)}
$$

with $\alpha \in(0,1)$.

We now show that for the case in which the economy is parameterized in this way and $S$ is a rectangular subset of $\mathbf{R}_{+}^{3}$, there are two types of equilibria, depending on the parameter values. For type 1 , the equilibrium consumption vector places mass one on some point $s_{1}=\left(c_{1}, h_{1}, k_{1}\right)$ that has $h_{1}>0$. Consequently, all agents work the same number of hours. For type 2 , the equilibrium consumption vector places mass on two points, $s_{0}$ and $s_{1}$. For $s_{0}$, the value of $h_{0}$ is zero. Thus, some fraction of the agents work $h_{1}>0$ hours and receive consumption $c_{1}$, while the remaining agents do not work (that is, $h_{0}=0$ ) and receive consumption $c_{0}$.

We start by describing the properties of solutions for the problem when $S$ is finite. We solve such finite-dimensional linear programs by using the simplex algorithm, which searches for optimal basic solutions. A basic solution is a feasible $\mathrm{x}$ that puts mass on a number of points equal to the number of constraints. Let $B \subseteq S$ be the set of these points and $a(s)=[1, c-f(k, 1) h, k]^{\prime}$ be the column vector defined by s. A basic solution also requires that the set of the column vectors defined by the basic solution, $\{a(s): s \in B\}$, be linearly independent. A basic solution is degenerate if the number of points with strictly positive mass is less than the number of constraints.

When the constraint set is closed, bounded, and nonempty, a solution exists. The dual constraints or first-order conditions with respect to $x_{8}$ of the finite-dimensional linear program are 


$$
\mathrm{U}(\mathrm{c}, \mathrm{h})-\mu_{0}-\mu_{1}[\mathrm{c}-\mathrm{f}(\mathrm{k}, 1) \mathrm{h}]-\mu_{2} \mathrm{k} \leq 0, \text { for all } \mathrm{s}=(\mathrm{c}, \mathrm{h}, \mathrm{k}) \in \mathrm{S}
$$

where $\mu_{0}, \mu_{1}$, and $\mu_{2}$ are the Lagrange multipliers associated with the constraints (16)-(18). Equation (19) must hold with equality if $x_{s}$ is strictly positive.

Numerical calculations deliver basic solutions for the finite-dimensional linear programs that are nondegenerate; that is, three points receive strictly positive mass. We, however, observe clusters: two or all three of the three points are close. If we choose progressively finer grids on $\mathrm{S}$, we observe that the points in a cluster converge. The basic solutions remain nondegenerate.

The convergence associated with the grid refinement is a convergence toward the solution of the linear programming problem where $S$ is no longer a finite set but a rectangular subset of $\mathbf{R}^{3}$. For this case, the summation is replaced by integration over a positive measure $\mathrm{x}$. The number of constraints remains finite. Consequently, we are dealing with a semi-infinite linear program.

Basic solutions for linear programs of this type are similar to solutions of finite-dimensional programs. An optimal measure $\mathrm{x}$ is atomic and assigns positive mass to no more points than there are constraints. All points in S continue to satisfy equation (19), and if a point receives positive mass, (19) holds with equality. This equality implies that any point $s_{i}=\left(c_{i}, h_{i}, k_{i}\right)$ that receives positive mass must maximize the left side of (19) with respect to s. For an introduction to semiinfinite linear programming, see Glashoff and Gustafson (1983).

We now show that there can be, at most, two points which receive positive mass; that is, the solution to the infinite-dimensional program is degenerate. We first note that, conditional on $\mathrm{k}$, the left side of (19) is a strictly concave function in $\mathrm{c}$ and $\mathrm{h}$ :

$$
\mathrm{V}(\mathrm{c}, \mathrm{h}, \mathrm{k}) \equiv \mu_{1} \mathrm{f}(\mathrm{k}, 1) \mathrm{h}+\mathrm{U}(\mathrm{c}, \mathrm{h})-\mu_{1} \mathrm{c}-\mu_{2} \mathrm{k}
$$

The solution to the problem of maximizing $V(c, h, k)$ by choice of $c$ and $h$ is 
(20) $\quad h *(k)= \begin{cases}1-\left[\frac{\mu_{1}}{\gamma}\right]^{-1 / \sigma}\left[\frac{\gamma}{(1-\gamma)} k^{\alpha}\right]^{-(\gamma \sigma+1-\gamma) / \sigma}, & k \geq \underline{k} \\ 0, & k<\underline{k}\end{cases}$

and

$$
\mathrm{c}^{*}(\mathrm{k})= \begin{cases}{\left[\frac{\mu_{1}}{\gamma}\right]^{-1 / \sigma}\left[\frac{\gamma}{(1-\gamma)} \mathrm{k}^{\alpha}\right]^{-(1-\gamma)(1 / \sigma-1)},} & \mathbf{k} \geq \underline{\mathrm{k}} \\ {\left[\frac{\mu_{1}}{\gamma}\right]^{-1 /(\gamma \sigma+1-\gamma)},} & \mathrm{k}<\underline{\mathrm{k}}\end{cases}
$$

where $\underline{\mathrm{k}}>\mathbf{0}$ depends on $\mu_{1}, \gamma$, and $\sigma$.

From the definition of the function $V$, it is immediate that $\left(c_{0}, h_{0}, k_{0}\right)=(c *(0), 0,0)$ is a local maximum of $\mathrm{V}$, given $\mu_{1}$ and $\mu_{2}$. Now let

$$
\mathrm{W}(\mathrm{k})=\mathrm{V}\left[\mathrm{c}^{*}(\mathrm{k}), \mathrm{h} *(\mathrm{k}), \mathrm{k}\right]
$$

The first and second derivatives of $\mathrm{W}$ with respect to $\mathrm{k}$, for $\mathbf{k}>\mathbf{k}$, are

$$
\begin{aligned}
& \frac{\partial \mathrm{W}}{\partial \mathrm{k}}=\alpha \mu_{1} \mathrm{k}^{(\alpha-1)} \mathrm{h}^{*}(\mathrm{k})-\mu_{2} \\
& \frac{\partial^{2} \mathrm{~W}}{\partial \mathrm{k}^{2}}=\alpha \mu_{1} \mathrm{k}^{(\alpha-2)}\left\{\alpha\left[\gamma+\frac{(1-\gamma)}{\sigma}\right]\left[1-\mathrm{h}^{*}(\mathrm{k})\right]-(1-\alpha) \mathrm{h}^{*}(\mathrm{k})\right\} .
\end{aligned}
$$

Since $h^{*}$ is a monotone increasing function of $k$, the second derivative of $W$ changes sign only once: it starts out positive for $\mathrm{k}=\underline{\mathrm{k}}\left(\mathrm{h}^{*} .=0\right)$ and then becomes negative. Thus, the first derivative of $\mathbf{W}$, which is negative at $\underline{\mathbf{k}}$, first increases and then decreases. There are then, at most, two values for which the first derivative is zero. If there are two, then only the larger is a local maximum. We do know that a global maximum exists at some $k_{1}>0$, since any solution involves some production of the consumption good. Therefore, at most, the function $\mathrm{W}$ has two local 
maxima, $k_{0}=0$ and $k_{1}>0$. To illustrate, we include a graph of the function $\mathrm{W}$, shown in Figure 1. The plot is for the parameter values $\alpha=0.3, \gamma=0.3, \sigma=0.8$, and $\overline{\mathrm{k}}=2.0$.

We now develop a sufficient condition that ensures the solution has positive mass on two points. The nature of the argument is to show that under this condition, a solution which puts mass on a single point leads to a contradiction.

PROPOSITION. If

$$
\alpha\left[\gamma+\frac{(1-\gamma)}{\sigma}\right](1-\gamma)-(1-\alpha) \gamma>0
$$

then the equilibrium places positive mass on two points.

Proof. Under the conjecture of all the mass being placed on a single point $s_{1}=\left(c_{1}, h_{1}, k_{1}\right), x_{31}=1$, the value of $k_{1}$ must be $\bar{k}$. After solving for $\mu_{1}$, (23) implies that the second derivative of $W$ is positive at $k=\bar{k}$, if and only if (24) is satisfied. The second derivative of $W$, being positive at $k_{1}$, contradicts $\mathbf{k}_{1}$ maximizing $\mathrm{W}$. But we have already shown that points receiving positive mass must maximize W. This establishes the result.

The result implies that for parameter values commonly used in real business cycle theory (for example, Hansen 1985) or public finance (for example, Auerbach and Kotlikoff 1987), we will have a type 2 solution, where not all agents will be working. If $\gamma \leq 2-1 / \alpha$, then inequality (24) holds for all $\sigma$. If $\gamma>2-1 / \alpha$, inequality (24) is satisfied for a sufficiently small $\sigma$. The intuition for the existence of type 2 solutions is that the gains in production, generated by splitting the population into two groups, more than compensate for the corresponding loss in expected utility due to the implied randomness in the agents' consumption. A sufficiently small risk aversion $\sigma$ ensures that this loss is not too large. 


\section{Existence of Competitive Equilibrium}

The economies discussed so far share the following structure: On the consumption side, there is a finite set $S$ with cardinality $q$. There is a finite number of agent types $i \in I$, and each agent type $i$ has preferences on $S$ represented by $U^{i}: S \rightarrow \mathbf{R}$. There is a continuum of measure $\lambda^{i}$ of each agent type $i$. The commodity space $L$ is the space of signed measures defined on the Borel $\sigma$-algebra of $S$. The consumption set of a type $i$ agent is defined as

$$
X^{i}=\left\{x \in L_{+}: \sum_{s \in S} x_{s}=1, \quad g^{i}(x) \leq 0\right\}
$$

where $\mathrm{g}^{\mathrm{i}}$ is a finite-dimensional linear mapping defined on $\mathrm{L}$. Preferences on consumption bundles are represented by the linear functional $u^{i}: X^{i} \rightarrow \mathbf{R}$ of the form

$$
\mathrm{u}^{\mathrm{i}}(\mathrm{x})=\sum_{\mathbf{s}} \mathrm{U}^{\mathrm{i}}(\mathrm{s}) \mathrm{x}_{\mathbf{s}}
$$

The production set is

$$
\mathrm{Y}=\left\{\mathrm{y} \in \mathrm{L}_{+}: \text {there exists } \mathrm{z} \in \mathbf{R}_{+}^{\ell} \text { for which } \mathrm{r}(\mathrm{x}, \mathrm{z}) \leq 0\right\}
$$

where $r$ is also a finite-dimensional linear mapping defined on $\mathbf{R}^{\mathbf{q}+\ell}$.

For the economy $\mathrm{E}$, a competitive equilibrium is a price system (linear functional) v: $\mathrm{L} \rightarrow \mathbf{R}$ and a feasible allocation $\left[\left(\mathrm{x}^{\mathrm{i}^{*}}\right)_{\mathrm{i} \in \mathrm{I}}, \mathrm{y}^{*}\right]$ such that

(i) for all i, $v\left(x^{i *}\right) \leq 0$, and for all $x \in X^{i}$ with $u^{i}(x)>u^{i}\left(x^{i *}\right), v(x)>0$

(ii) $y \in Y$ implies $v(y) \leq v\left(y^{*}\right)$.

We consider competitive equilibrium allocations that are anonymous. For these allocations an agent's consumption bundle or contract depends only on that agent's type. A more general formulation would allow for the possibility that agents of the same type receive different contracts. Below we first argue that theory imposes restrictions only on the realized distribution of consumption 
allocations. We then show that given any possible competitive equilibrium allocation, there will be an anonymous competitive equilibrium with the same realized distribution of consumption allocations. Thus the restriction to anonymous competitive equilibrium allocations is not binding with respect to the realized distribution of consumption allocations, and it is this distribution that is to be explained.

When we confront our model economies with empirical observations, we treat lottery contracts in the same way we treat the Walrasian auctioneer in competitive economic theory. There, we do not expect to have observations of the auctioneer's actions in clearing markets; rather, the auctioneer is used as an "as-if" construct. The observed consumption allocations, however, are interpreted as the equilibrium outcomes of an economy in which an auctioneer clears all markets. Here, we treat contracts analogously. We do not expect to make observations on actual contracts, but we do expect to make observations on realized distributions of consumption allocations for different agent types. To evaluate the model, we are then limited to its implications for these distributions. Our point is that, given this interpretation, the two formulations are observationally equivalent.

Let each agent be indexed by a name e $\in \mathrm{E}=[0, \lambda]$, and let $\mu$ be the Lebesgue measure. (This approach follows Hildenbrand 1974.) Let h: $\mathrm{E} \rightarrow \mathrm{I}$ be some Borel-measurable function that assigns each agent to some type. The function $h$ is such that for the set of agents of each type $i$, expressed as

$$
E^{i}=\{e \in E: h(e)=i\}
$$

the measure of $E^{i}$ is $\lambda^{i}$.

Let $\phi(v, i)$ be the demand correspondence of a type $i$ agent. Since agent $e$ is of type $h(e)$, the demand correspondence of this agent is $\phi(v, h(e))$. Using this notation, we define a competitive 
equilibrium allocation as a price $\mathrm{v}^{*}$; an integrable function $x^{*}: \mathrm{E} \rightarrow \mathrm{I}$ satisfying $\mathrm{x}^{*} \in \phi\left(\mathrm{v}^{*}, \mathrm{~h}(\mathrm{e})\right)$, for all $\mathrm{e} \in \mathrm{E}$; and a profit-maximizing production plan $\mathrm{y}^{*} \in \mathrm{Y}$ such that $\int_{\mathrm{E}} \mathrm{x}^{*}(\mathrm{e}) \mu(\mathrm{de})=\mathrm{y}^{*}$.

Given a competitive equilibrium, we construct an anonymous competitive equilibrium with the same realized distribution on consumption allocations for each agent type as follows. Let

$$
\overline{\mathrm{X}}^{\mathrm{i}} \equiv \int_{\mathbf{B}^{\mathrm{i}}} \mathrm{x}^{*}(\mathrm{e}) \mu(\mathrm{de}) / \lambda^{\mathrm{i}}
$$

The measure $\overline{\mathbf{x}}^{i}$ defined above is a probability measure on consumption allocations. It is not the measure for a particular agent $e$, but it is a probability measure conditional on the fact that we have an agent $e$ of type $i$ holding an equilibrium contract $x^{*}(e)$.

Since the demand correspondence is convex valued, then $\bar{x}^{i}$, which is a convex combination of elements belonging to $\phi\left(\mathrm{v}^{*}, \mathrm{i}\right)$, also belongs to that set. That is, $\overline{\mathrm{x}}^{\mathrm{i}}$ is optimal given $\mathrm{v}^{*}$. Point $\mathrm{y}^{*}$ is profit maximizing, given $\mathbf{v}^{*}$.

We still need to show that the anonymous allocation $\left[\left(\overline{\mathrm{x}}^{\mathrm{j}}\right), \mathrm{y}^{*}\right]$ is market clearing. Given the definition of $\overline{\mathbf{x}}^{\mathbf{i}}$,

$$
\sum_{i} \lambda^{i} \bar{x}^{i}=\sum_{i} \int_{\mathbf{E}^{i}} x^{*}(e) \mu(\mathrm{de})=y^{*}
$$

This establishes that the anonymous allocation $\left[\left(\overline{\mathrm{x}}^{\mathrm{i}}\right), \mathrm{y}^{*}\right]$ and the price system $\mathrm{v}^{*}$ are an anonymous competitive equilibrium.

What we have just defined is a mapping from the set of competitive equilibria into the set of anonymous competitive equilibria. Given this mapping, we can group competitive equilibrium allocations into equivalence classes indexed by their implied anonymous competitive equilibrium allocation. 
Since we assume that a law of large numbers holds, the realized distribution on consumption allocations for an agent type $i$ coincides with the probability measure $\bar{x}^{i}$. This holds for all competitive allocations. Therefore, all competitive equilibrium allocations within an equivalence class and their corresponding anonymous competitive equilibrium allocation do, in this sense, have these same observational implications. The two equilibrium formulations are observationally equivalent, if we have data on the realized outcomes and not the contracts themselves. Typically, we deduce the nature of the equilibrium contract from the realized distributions of actual consumption allocations.

To prove existence of a competitive equilibrium, we slightly modify McKenzie's (1959, 1981) proof. The first modification is a matter of interpretation. McKenzie assumes a finite number of agents, but we interpret his agent $i$ as the agent type $i$. For our problems we show that all of McKenzie's assumptions that guarantee existence of an equilibrium are satisfied, except for Assumption 4. We also show that for our problems Assumption 4 can be dispensed with.

ASSUMPTION 1. $\mathrm{X}^{\mathrm{i}}$ is convex, closed, and bounded from below.

From the definition of the consumption possibility set, it follows that $X^{i}$ is a closed convex subset of the unit simplex. Thus, $X^{i}$ not only satisfies Assumption 1 but is also compact.

ASSUMPTION 2. $\mathrm{X}^{\mathrm{i}}$ is completely ordered by a convex and closed preference relation.

In the span-of-control economy, preference relations are represented by linear functions on $\mathrm{X}^{\mathrm{i}}$ and, therefore, are continuous and convex.

Assumption 3. $\mathbf{Y} \subseteq \mathbf{R}_{+}^{q}$ is a closed convex cone.

The assumption follows from the definition of $\mathrm{Y}$. 
ASSUMPTION 4. $Y \cap \mathbf{R}_{+}^{q}=\{0\}$.

This assumption states that null is the only joint element of the production set and the positive orthant. For our economy, however, the production set is actually a subset of the positive orthant. We can still use McKenzie's proof since Assumption 4 is used only to prove compactness of the set of feasible allocations. For our application, the compactness of this set follows trivially from our additional assumption that the consumption possibility set for each agent type is compact, together with Assumption 3.

The appropriate assumptions concerning the relation between the production and consumption sides are also satisfied. Let $X=\Sigma_{i} \lambda \lambda^{i} x^{i}$, and let $I^{\prime}, I^{\prime \prime}$ be nonempty subsets of $I$ such that $I^{\prime} \cap$ $I^{\prime \prime}=\phi$ and $I^{\prime} \cup I^{\prime \prime}=I$. Then define $X^{I^{\prime}}=\Sigma_{i \in I^{\prime}} \lambda^{i} X^{i}$ and $X^{I^{\prime \prime}}=\Sigma_{i \in I^{\prime}} \lambda^{i} X^{i}$.

Assumption 5. $X^{i} \cap Y \neq \phi$ for all i. Moreover, there is a common point $\bar{x}$ in the relative interiors of $\mathrm{X}$ and $\mathrm{Y}$.

The commodity point $x$, which puts unit mass on the event $s=\left(0, k^{i}, m^{i}\right)$, is a joint element of $\mathrm{X}^{\mathrm{i}}$ and $\mathrm{Y}$. A point $\overline{\mathrm{X}}$ that is in the relative interior of $\mathrm{X}$ and in the relative interior of $\mathrm{Y}$ can be constructed as follows. For each agent type $i$, choose a point $x^{i}$ that puts equal mass on each set $S_{\mathrm{km}}^{i}=\left\{s^{\prime}=\left(c^{\prime}, k^{\prime}, m^{\prime}\right) \in S^{i}: k^{\prime}=k, m^{\prime}=m\right\}$, where $k \in K$ and $k \leq k^{i}$ and $m \in\left\{0, m^{i}\right\}$. This implies that certain quantities of labor, capital, and managers of each type are available for production. Now distribute labor and capital equally among all available managers. This distribution implies that a certain quantity of the consumption good will be produced. So far, only the marginal distribution of $x$ on $S$ with respect to $h$ and $m$ has been determined. Within each set $S_{\mathrm{km}}^{i}$, assign equal mass to each point. There is then a unique value for that mass such that total production and 
total consumption of the consumption good are equal. We now have a point $\overline{\mathrm{X}} \in \mathrm{X} \cap \mathrm{Y}$ such that $\bar{x}_{s}>0$ for all $s \in U_{i \in S} S^{i}$.

Let $H_{y}=\left\{x \in R^{q}: x_{s}=0\right.$ if $\left.s \notin U_{i \in I} S^{i}\right\}$. Point $\bar{x}$ is in the nonempty interior of $Y$ relative to $H_{y}$. Let $H_{x}=\left\{x \in H_{y}: \Sigma_{z} x_{z}=1\right\}$. Point $\bar{x}$ is in the nonempty interior of $X$ relative to $H_{x}$.

Assumption 6. However $\mathrm{I}^{\prime}$ and $\mathrm{I}^{n}$ may be selected, if $\mathrm{x}^{\mathrm{I}^{\prime}} \in \mathrm{Y}-\mathrm{X}^{\mathrm{I}^{\prime \prime}}$, then there is also $\mathrm{w} \in \mathrm{Y}-$ $\mathrm{X}^{\mathrm{I}^{\prime \prime}}$ such that $\mathrm{w}=\overline{\mathrm{x}}^{\mathrm{I}^{\prime}}-\mathrm{x}^{\mathrm{l}^{\prime}}$ and $\mathrm{u}\left(\overline{\mathrm{x}}^{\mathrm{i}}\right) \geq \mathrm{u}\left(\mathrm{x}^{\mathrm{i}}\right)$ for all $\mathrm{i} \in \mathrm{I}^{\prime}$, and $\mathrm{u}\left(\overline{\mathrm{x}}^{i}\right)>\mathrm{u}\left(\mathrm{x}^{\mathrm{i}}\right)$ for some $\mathrm{i} \in \mathrm{I}^{\prime}$.

This assumption is satisfied if $\mathrm{C}$ contains an element $\mathrm{c}_{\max }$ that cannot be produced, given the endowment of capital and labor. In this case, preferences satisfy local and global nonsatiation with respect to feasible allocations.

Given that these five assumptions are satisfied, by Theorem 1 of McKenzie (1959), a competitive equilibrium exists for the economy $\mathrm{E}$.

We can relax the restriction that $S$ is a finite set and allow it to be a compact subset of a finite-dimensional Euclidean space. Then the commodity space $L$ becomes the space of signed measures on the Borel $\sigma$-algebra of these sets, $M(S)$. The subset of feasible allocations is compact with respect to the weak* topology since these allocations are probability measures and therefore uniformly bounded.

To show that a competitive equilibrium for such an economy exists, we study competitive equilibria for a sequence of economies. We show that the competitive equilibrium allocations and prices converge to an allocation and price system that is a competitive equilibrium for the economy with commodity space $\mathrm{M}(\mathbf{S})$. The proof follows Mas-Colell (1975) and Prescott and Townsend (1984), and proceeds in three steps. First, we construct a sequence of economies and show that their competitive equilibrium allocations converge to a limit allocation that is feasible for the economy with commodity space $M(S)$. Second, we prove the existence of a continuous price function $v$ and 
a cheaper point at prices $\mathrm{v}$ for each agent type in the limit economy. In the third and last step we use the results from the second step to establish that the limit allocation is optimal at the limit price system v.

We consider sequences of economies where, for the $\mathrm{j}^{\text {th }}$ economy, we restrict the commodity space $L_{j}=M\left(S_{j}\right)$ to a set of atomic signed measures that have mass only on a finite number of points, $S_{j} \subseteq S$. Let $D$ be a countable, everywhere dense subset of $S$ and let $S_{j}$ contain the first $j$ elements of $D$ and the set $\left\{\left(c_{\max }, k^{i}, m^{i}\right): i \in I\right\}$. Then $S_{j} \rightarrow S$ in closed convergence. Similarly, for the production possibility set we restrict the space of activity measures to atomic signed measures that have mass only on a finite number of points, $A_{j} \subseteq A$, and $A_{j} \rightarrow A$ in closed convergence.

For each of the economies $E_{j}$, there exist a competitive equilibrium allocation and price system, $\left[\left(\bar{x}_{j}\right)_{i \in I}, \bar{y}_{j}\right]$ and $v_{j}$, as already shown. Since the sequence of equilibrium allocations is a subset of a weak* compact set, the sequence has a convergent subsequence. And again, the limit of this subsequence of competitive equilibrium allocations, $\left[\left(\overline{\mathrm{x}}^{\mathrm{i}}\right)_{\mathrm{i}} \in \mathbf{I}, \overline{\mathrm{y}}\right]$, is a feasible allocation for the economy with an unrestricted commodity space $\mathrm{L}$. This completes the first step of the proof.

The second step of the argument is specific to our economy and concerns the existence of a continuous price function $v$ and the existence of a cheaper point at prices $v$ for each agent type in the limit economy.

Lemma 1. There exists a subsequence of equilibrium prices $\mathbf{v}_{j}$ that converges uniformly to a continuous function $\mathrm{v}$ on $\mathrm{S}$.

Proof. We first show that we can define equilibrium prices $\mathbf{v}_{\mathbf{j}}$ as a linear function on $\mathrm{S}_{\mathrm{j}}$. This property is then used to prove the existence of a limit price function $\mathbf{v}$ that is continuous on $\mathrm{S}$.

The equilibrium price function for the $\mathrm{j}^{\text {th }}$ economy is constructed from the firm's optimality conditions. These conditions are 


$$
\begin{aligned}
& \mathrm{v}_{\mathrm{sj}}-\mu_{1 \mathrm{j}} \mathrm{c}+\mu_{2 \mathrm{j}} \mathrm{k}+\mu_{3 \mathrm{mj}} \leq 0, \quad \text { for all } \mathrm{s}=(\mathrm{c}, \mathrm{k}, \mathrm{m}) \in \mathrm{S}_{\mathrm{j}} \\
& \mu_{1 \mathrm{j}} \mathrm{mg}[\mathrm{f}(\mathrm{k}, \mathrm{n})]-\mu_{2 \mathrm{j}} \mathrm{k}-\mu_{3 \mathrm{mj}}-\mu_{30 \mathrm{j}} \mathrm{n} \leq 0, \quad \text { for all } \mathrm{a}=(\mathrm{k}, \mathrm{m}, \mathrm{n}) \in \mathrm{A}_{\mathrm{j}}
\end{aligned}
$$

where $\mu_{1}, \mu_{2}$, and $\mu_{3 \mathrm{~m}}$ are the Lagrange multipliers associated with constraints (3)-(6).

For each economy $j$ there is a competitive equilibrium with a price system $v_{j}$ such that (25) is binding for all $s \in S_{\mathrm{j}}$. To see that such an equilibrium exists, take any competitive equilibrium for the $j^{\text {th }}$ economy. If for this equilibrium (25) is not binding for some $s^{\prime} \in S_{j}$, then $s^{\prime}$ is not produced and its price is below the supply reservation price. In this case the price for $\mathbf{s}^{\prime}$ can be raised until (25) is binding. The competitive equilibrium allocation continues to be optimal at the higher price. The firm's optimality conditions are satisfied, and since agents did not consume s' at the original price they will not consume $\mathbf{s}^{\prime}$ now at higher prices.

The sequence of competitive equilibria associated with the sequence of economies is chosen from the class of equilibria with (25) binding. Equation (25) thereby defines the price system $v_{j}$ as a linear function on S. Given this representation of the price system $v_{j}$, there is a subsequence of equilibrium prices that converges uniformly to a continuous function $v$ on $S$, if there exists a converging subsequence for the Lagrange multipliers $\mu_{\mathrm{j}}$.

We first show that $\mu_{1 \mathrm{j}}>0$. Suppose $\mu_{1 \mathrm{j}}=0$. Then from the definition of prices, it follows that $\mathrm{v}_{\mathrm{sj}} \leq 0$ for all $\mathrm{s} \in \mathrm{S}_{\mathrm{j}}$. Now an agent of type i solves the following problem in the $\mathrm{j}^{\text {th }}$ economy:

$$
\max _{x \geq 0} \sum_{s \in S_{j}^{i}} U(c) x_{s}
$$

subject to constraints

$$
\begin{aligned}
& \sum_{s \in S_{i}^{i}} v_{s j} x_{s} \leq 0 \\
& \sum_{s \in S_{j}^{i}} x_{s}=1 .
\end{aligned}
$$


If $v_{\mathrm{sj}} \leq 0$ for all $s$ in $S_{j}^{i}$, then each agent will put mass only on points $s$ with $c=c_{\max }$ as $U$ is monotone increasing in $c$. But $c_{\max }$ cannot be produced for all agents of any type; thus, the allocation cannot be feasible. Therefore, $\mu_{\mathrm{ij}}>0$.

Prices of a competitive equilibrium can be normalized arbitrarily. Since equations (25) and (26) are jointly linear in prices $v_{j}$ and multipliers $\mu_{j}$, multipliers can be normalized arbitrarily. The Lagrange multipliers are nonnegative and we have shown that for the $\mathrm{j}^{\text {th }}$ economy $\mu_{1 \mathrm{j}}>0$. Therefore, it is possible to normalize $\mu_{\mathrm{j}}$ so that $\left|\mu_{\mathrm{j}}\right|=1$. This, in turn, ensures the existence of a converging subsequence $\mu_{\mathrm{j}} \rightarrow \mu$. Given our definition of the price system in equation (25), the limit price function $\mathbf{v}$ is continuous on $\mathrm{S}$ and the subsequence of price systems converges uniformly. This completes the proof.

To show that the limit allocation is utility maximizing at the limit prices, we now prove the existence of a cheaper point for each type.

Lemma 2. For each agent type $\mathrm{i}$, there is a feasible choice $\mathrm{x}_{0} \in \mathrm{X}^{\mathrm{i}}$ such that $\mathrm{v}\left(\mathrm{x}_{0}\right)<0$.

Proof. We show that for the converging subsequence of Lagrange multipliers, $\mu_{\mathrm{j}} \rightarrow \mu$ (developed in Lemma 1), the limit of $\mu_{1 \mathrm{j}}$ is strictly positive. This implies that $\mu_{3 \mathrm{~m}}>0$ for all $\mathrm{m} \in \mathrm{M}$, which guarantees the existence of a cheaper point.

Suppose that $\mu_{1 j} \rightarrow 0$. If, in addition, $\mu_{2 j} \rightarrow 0$, then there is at least one $\mathrm{m}$ such that $\mu_{3 \mathrm{mj}} \rightarrow \mu_{3 \mathrm{~m}}$ and $\mu_{3 \mathrm{~m}}>0$, since $\left|\mu_{\mathrm{j}}\right|=1$. But then the measure $\mathrm{x}_{\max }$, which puts unit mass on the point $\left(c_{\max }, k^{i}, m^{i}\right)$, will be in the budget set for agents with the ability $m^{i}=m$ for some economy $j$. The agents of this type will choose the measure, but the choice is not feasible. Therefore, $\mu_{2}>0$ if $\mu_{1}=0$. But if $\mu_{2}>0$, the same $x_{\max }$ is again in the budget set for some agent type in some economy j. Thus, $\mu_{1}$ must be strictly positive. 
It follows from (26) that $\mu_{3 \mathrm{~m}}>0$ for all $\mathrm{m} \in \mathrm{M}$ if $\mu_{1}>0$. To see this, rewrite (26) as follows

$$
\mu_{3 \mathrm{~m}} \geq \mu_{1} \mathrm{mg}[\mathrm{f}(\mathrm{k}, \mathrm{n})]-\mu_{2} \mathrm{k}-\mu_{30} \mathrm{n}, \text { for all } \mathrm{a} \in \mathrm{A} \text {. }
$$

The maximum of the right side of (27) is greater than or equal to zero. Since by assumption $\mathrm{g}^{\prime}(0)=\infty$ and since $\mu_{1}>0$, the maximum of the right side attains a value strictly greater than zero. Therefore, $\mu_{3 \mathrm{~m}}>0$ for all $\mathrm{m} \in \mathrm{M}$.

Each agent type is endowed with some managerial ability $m \in M$. Therefore, the measure $\mathrm{x}_{0}$ that puts unit mass on the point $\mathrm{s}=(0,0, \mathrm{~m})$ is feasible for that agent. But since $\mu_{3 \mathrm{~m}}>0$, the value of this bundle is negative; that is, $v\left(x_{0}\right)<0$. This completes the proof of Lemma 2 .

For the third and final part of the argument we now use Lemmas 1 and 2 to establish that the limit allocation is optimal at the limit price system. We first discuss the problems facing the households. Since the analysis applies to all agent types, we simplify the notation by dropping the agent type superscript.

Suppose that the consumption point $\overline{\mathrm{x}}$ is not optimal at the price system $\mathrm{v}$; that is, suppose there exists an $x$ such that $x \in X, u(x)>u(\bar{x})$, and $v(x) \leq v(\bar{x})=0$. We distinguish two cases, $v(x)<v(\bar{x})$ and $v(x)=v(\bar{x})$. We establish that the first case leads to a contradiction. We then show that the second case implies that there is some $x^{t} \in X$ such that $u\left(x^{t}\right)>u(\bar{x})$ and $v\left(x^{t}\right)<v(\bar{x})$. But the existence of such a point leads to a contradiction, as already established for the first case.

Suppose that the first case is true; that is, suppose $v(x)<v(\bar{x})$. As $S_{j} \rightarrow S$ in closed convergence and $S$ is compact, we can find a sequence of allocations $\left\{x_{j}\right\}$ such that $x_{j} \in X_{j}$ and $x_{j} \rightarrow x$. (See Fact 6 of Mas-Colell 1975, p. 274.) But then $v_{j}\left(x_{j}\right) \rightarrow v(x)$ and $v_{j}\left(\bar{x}_{j}\right) \rightarrow v(\bar{x})$, since the measures $x$ are bounded and $v_{j}$ converges uniformly. In addition, $u\left(x_{j}\right) \rightarrow u(x)$ and $u\left(\bar{x}_{j}\right) \rightarrow u(\bar{x})$. 
This means that there is some economy $E_{j}$ for which $v_{j}\left(x_{j}\right)<v_{j}\left(\bar{x}_{j}\right)$ and $u\left(x_{j}\right)>u\left(\bar{x}_{j}\right)$. This contradicts $\bar{x}_{j}$ being optimal at the price system $\mathbf{v}_{\mathbf{j}}$.

Alternatively, suppose that the second case is true; that is, suppose $v(x)=v(\bar{x})$. We have already shown that there is a cheaper point for each agent type. Let $x_{0} \in X$ with $v\left(x_{0}\right)<0$ be such a point. Define points $x^{t}=(1-t) x+t x_{0}$ with $t \in[0,1]$. Then $x^{t} \in X, v\left(x^{t}\right)<v(\bar{x})$ for $t>0$ and $u\left(x^{t}\right)>u(\bar{x})$ for $t$ sufficiently small. But this means that $x^{t}$ satisfies the conditions of the first case, and we have already established that this leads to a contradiction.

The argument proving that the limit allocation is optimal for the firm is similar. Suppose $\bar{y}$ is not optimal for the firm; that is, suppose there exists some $y \in Y$ such that $v(y)>v(\bar{y})$. We can find a sequence $\left\{y_{j}\right\}$ such that $y_{j} \in Y_{j}$ and $y_{j} \rightarrow y$. But then $v_{j}\left(y_{j}\right) \rightarrow v(y)$ and $v\left(\bar{y}_{j}\right) \rightarrow v(\bar{y})$. This implies that there is some economy $E_{j}$ such that $v_{j}\left(y_{j}\right)>v_{j}\left(\bar{y}_{j}\right)$, which contradicts $\bar{y}_{j}$ being optimal at the price system $v_{j}$. 


\section{Footnotes}

${ }^{1}$ The fact that we restrict allocations to ones that treat agents of the same type in the same way is discussed in Section 3.

${ }^{2}$ In his study of executive compensation, Tuck (1954) deals with managerial span-of-control in an informal but general equilibrium way. We thank Lionel McKenzie for bringing this work to our attention.

${ }^{3}$ Although it is possible to define a lower-dimensional commodity space for this example, it is not desirable. By considering the more general space, results such as the convexity of $Y$ are transparent. 


\section{References}

Arrow, Kenneth J., and Debreu, Gerard. 1954. Existence of an equilibrium for a competitive economy. Econometrica 22, no. 3 (July): 265-90.

Auerbach, Alan J., and Kotlikoff, Laurence J. 1987. Dynamic fiscal policy. Cambridge: Cambridge University Press.

Glashoff, Klaus, and Gustafson, Sven-Åke. 1983. Linear optimization and approximation. New York: Springer.

Hansen, Gary D. 1985. Indivisible labor and the business cycle. Journal of Monetary Economics 16 , no. 3 (November): $309-27$.

Hildenbrand, Werner. 1974. Core and equilibria of a large economy. Princeton: Princeton University Press.

Lucas, Robert E., Jr. 1978. On the size distribution of business firms. Bell Journal of Economics 9, no. 2 (Autumn): 508-23.

Mas-Colell, Andreu. 1975. A model of equilibrium with differentiated commodities. Journal of Mathematical Economics 2 (June-September): 263-95.

McKenzie, Lionel W. 1959. On the existence of general equilibrium for a competitive market. Econometrica 27, no. 1 (January): 54-71.

- 1981. The classical theorem on existence of competitive equilibrium. Econometrica 49, no. 4 (July): 819-41.

Prescott, Edward C., and Townsend, Robert M. 1984. General competitive analysis in an economy with private information. International Economic Review 25, no. 1 (February): 1-20.

Rogerson, Richard. 1988. Indivisible labor, lotteries and equilibrium. Journal of Monetary Economics 21, no. 1 (January): 3-16. 
Tuck, R. H. 1954. An essay on the economic theory of rank. Oxford: Basil Blackwell.

Uhlig, Harald. 1988. A law of large numbers for large economies. Research Department Working Paper 342, Federal Reserve Bank of Minneapolis. 


\section{Figure 1}

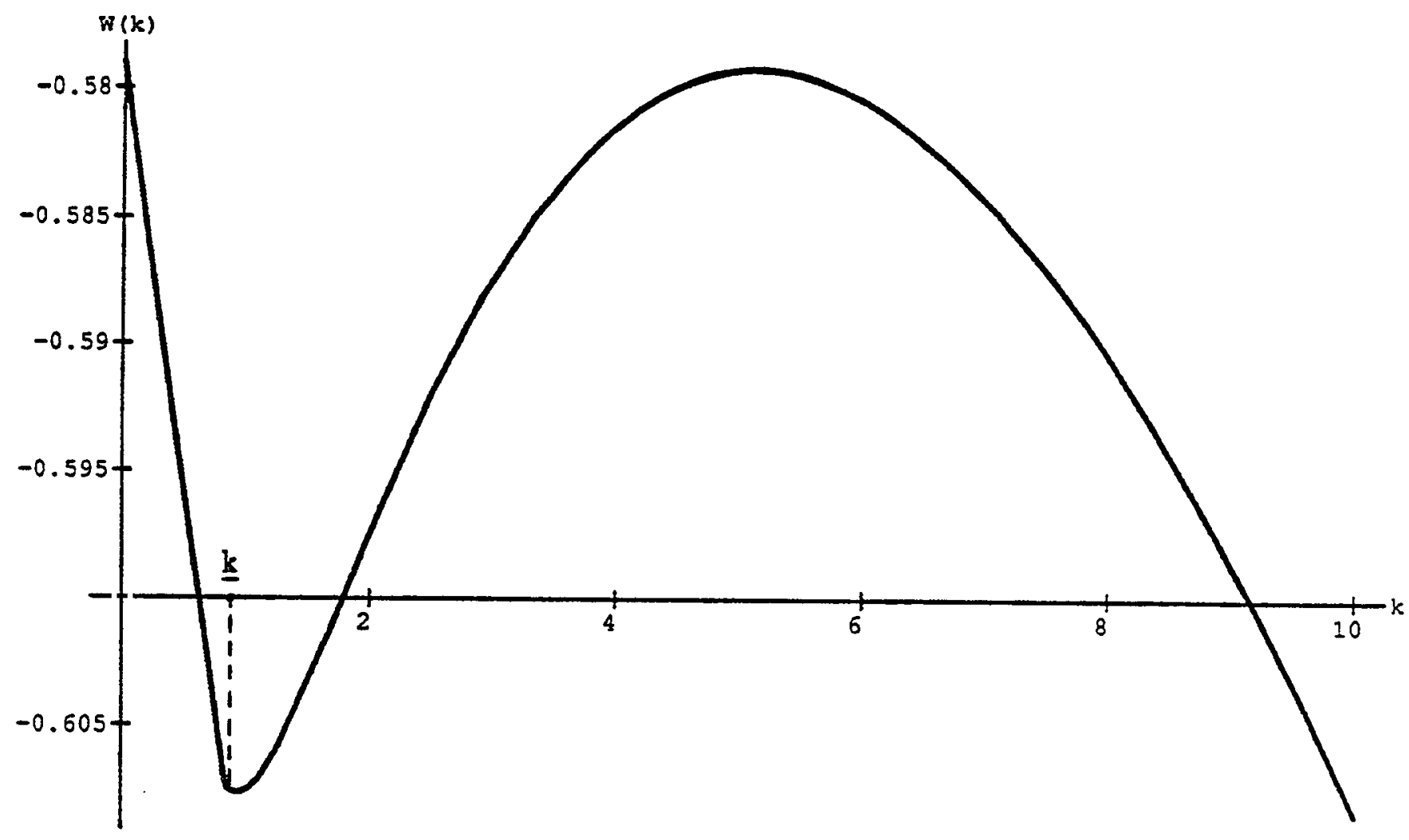

Parameter Values: $\alpha=0.3, \gamma=0.3, \sigma=0.8, \overline{\mathrm{k}}=2.0$ 